Abstract

\title{
Basin Entropy: A New Method to Measure Unpredictability in Physical Systems ${ }^{\dagger}$
}

\author{
Miguel A. F. Sanjuán \\ Nonlinear Dynamics, Chaos and Complex Systems Group, Departamento de Física, Universidad Rey Juan \\ Carlos, Madrid, Spain \\ + Presented at the Entropy 2021: The Scientific Tool of the 21st Century, 5-7 May 2021; Available online: \\ https://sciforum.net/conference/Entropy2021/.
}

Published: 5 May 2021

In nonlinear dynamics, basins of attraction are defined as the set of points that, taken as initial conditions, lead the system to a specific attractor. This notion appears in a broad range of applications where multistability is present, which is a common situation in neuroscience, economy, astronomy, ecology, and other disciplines. Nonlinear systems often give rise to fractal boundaries in phase space, hindering predictability. When a single boundary separates three or more different basins of attraction, we call them Wada basins. Usually, Wada basins have been considered even more unpredictable than fractal basins. However, this particular unpredictability has not been fully unveiled until the introduction of the concept of basin entropy. The basin entropy provides a quantitative measure of how unpredictable a basin is. With the help of several paradigmatic dynamical systems, we illustrate how to identify the ingredients that hinder the prediction of the final state. The basin entropy together with two new tests of the Wada property have been applied to some physical systems such as experiments of chaotic scattering of cold atoms, models of shadows of binary black holes, and classical and relativistic chaotic scattering associated to the Hénon-Heiles Hamiltonian system in astrophysics.

\section{References}

1. Daza, A.; Wagemakers, A.; Sanjuán, M.A.; Yorke, J.A. Testing for basins of Wada. Sci. Rep. 2015, 5, 16579.

2. Daza, A.; Wagemakers, A.; Sanjuán, M.A. Wada property in systems with delay. Commun. Nonlinear Sci. Numer. Simul. 2017, 43, 220-226.

3. Daza, A.; Wagemakers, A.; Georgeot, B.; Guéry-Odelin, D.; Sanjuán, M.A. Basin entropy: a new tool to analyze uncertainty in dynamical systems. Sci. Rep. 2016, 6, 31416.

4. Daza, A.; Georgeot, B.; Guéry-Odelin, D.; Wagemakers, A.; Sanjuán, M.A. Chaotic dynamics and fractal structures in experiments with cold atoms. Phys. Rev. A 2017, 95, 013629.

5. Daza, A.; Wagemakers, A.; Georgeot, B.; Guéry-Odelin, D.; Sanjuán, M.A. Basin Entropy, a Measure of Final State Unpredictability and Its Application to the Chaotic Scattering of Cold Atoms. In Chaotic, Fractional, and Complex Dynamics: New Insights and Perspectives; Springer: Cham, Switzerland, 2018.

6. Daza, A.; Shipley, J.O.; Dolan, S.R.; Sanjuán, M.A. Wada structures in a binary black hole system. Phys. Rev. D 2018, 98, 084050.

7. Daza, A.; Wagemakers, A.; Sanjuán, M.A. Ascertaining when a basin is Wada: the merging method. Sci. Rep. 2018, 8, 9954.

8. Wagemakers, A.; Daza, A.; Sanjuán, M.A. The saddle-straddle method to test for Wada basins. Commun. Nonlinear Sci. Numer. Simul. 2020, 84, 105167.

(C) 2021 by the authors. Licensee MDPI, Basel, Switzerland. This article is an open access article distributed under the terms and conditions of the Creative Commons Attribution (CC BY) license (http://creativecommons.org/licenses/by/4.0/). 